AUTHOR'S ABSTRACT OF THIS PAPER ISSUED BY

THE BIBLIOGRAPHIC SERVICE, NOVEMBER 10.

\title{
ON THE MORPHOGENESIS OF THE PAPILLA FOLIATA OF THE RABBIT
}

\author{
G. CARL HUBER AND ARNOLD H. EGGERTH \\ Department of Anatomy, University of Michigan
}

SIX FIGURES

There exist in the literature numerous contributions dealing with the form and distribution of the gustatory papillae in vertebrates and with the structure and innervation of the gustatory buds. This literature is foreign to the problem here under discussion and will not receive consideration at the present time. Relatively few observers have dealt with the development of the gustatory papillae, and fewer still have considered the development of the papilla foliata of the rabbit or of other forms in which this type of gustatory papilla is prominent. v. Wyss ${ }^{1}$ who was the first to call attention to the details of structure of the papilla foliata of the rabbit and the great number of gustatory buds there found, makes only incidental mention of its development, stating that it is quite well formed in the new born rabbit and contains taste buds which have, however, a more rounded form and are smaller than in the grown animal. Lustig ${ }^{2}$ states that with the aid of a lens he was able to recognize the papilla foliata in rabbit embryos having a length of $6 \mathrm{~mm}$. to $7.5 \mathrm{~mm}$. and that they are more developed in $10 \mathrm{~mm}$. rabbit embryos, however, that it was not possible for him so define the taste buds. A fairly comprehensive account of the development of the papilla foliata of the rabbit is given by Hermann. ${ }^{3}$ This observer found that

\footnotetext{
${ }^{1}$ v. Wyss. H., Die becherförmigen Organe der Zunge. Arch. f. Mik. Anat., vol. 6,1870 .

${ }^{2}$ Lustig, A., Beiträge zur Kenntniss der Entwickelung der Geschmacksknospen. Sitzungsber. Akad. d. Wissensch, Viena, Math. Nat. Classe, vol. 89, 1884.

${ }^{3}$ Harmann, F., Beitrag zur Entwicklungsgeschichte des Geschmacksorgans beim Kaninchen. Arch. f. Mik. Anat., vol. 24, 1885.
} 
the papilla foliata was first recognizable in rabbit embryos having a length of $54 \mathrm{~mm}$. (age, about twenty-three days), as an oval, slightly elevated area, with long axis horizontal and with faint grooves having a vertical direction. In longitudinal sections, papillae formed by simple infolding of the epithelium were observed. In embryos having a length of $70 \mathrm{~mm}$. (about twentyfour days) the foliate area is no longer quite horizontal in position, its posterior end being nearer the median line. The epithelial papillae are longer and broader, the primary infoldings presenting at about the middle of their depth, on each side, a slight projection, most fully developed in the middle of the area. In embryos having a length of $95 \mathrm{~mm}$., thus only a few days before birth the slight projectings of the primary folds have developed into secondary folds and the anlagen of the serous lingual glands are evident. In the new born rabbit the secondary folds of the papillae are longer, the whole area, however, presenting essentially the same appearance as that found in a $95 \mathrm{~mm}$. rabbit. In its main feature, we confirm the observations of Hermann. As a result of a comparative study Brücher ${ }^{4}$ concludes that the prototype of the gustatory papillae is the papilla fungiformis, out of which developed both the p. vallata and the p. foliata, since transition forms are observed. Gmelin ${ }^{5}$ on the other hand, also as a result of a comparative study, concludes that the p. vallata is not developed from the p. fungiformis, nor the $p$. foliata from the p. vallata, but that both $p$. vallata and p. foliata are independent in their origin, each having its special seat of development, and that transition forms are not observed. Hoffmann, ${ }^{6}$ Tuckermann, ${ }^{7}$ and more recently, Gråberg, ${ }^{8}$ have pub-

${ }^{4}$ Brücher, Über die Vertheilung und Anordnung der Geachmacks-papillen auf der Zunge der Säugethiere, spec. der Hufthiere. Deutsch. Zeitsch. f. Thiermed, u, verg. Path., vol. 10, 1884.

${ }^{5}$ Gmelin, Zur Morphologie der Papilla vallata und foliata. Arch. f. Mik. Anat., vol. 40, 1892.

${ }^{6}$ Hoffmann, Über die Verbreitung der Geschmacksorgane beim Menschen. Arch. f. Path. vol., 62, 1875.

7 Tuckermann, F., On the development of the taste-organs of man. Jour. Anat. Phys., vol. 23, 1889.

${ }^{8}$ Gråberg, J., Beiträge zur Genese der Geschmacksorgane des Menschen. Morphol. Arbeit,, vol. 8, 1898. 
lished concerning the development of the vallata papillae of man, but since their observations bear only incidentally on those here recorded their publications will not receive review here. M. Heidenhain, ${ }^{9,10}$ has in two relatively recent communications considered very fully certain aspects of the papilla foliata of the rabbit. Certain points discussed is these articles may here be touched on. He finds the foliata area of the grown rabbit to be of pear or egg-shape, with the narrower end directed caudally, the rounder end forwards. The folds, of which there are about 16, run from the ventral to the dorsal border, diverging somewhat dorsally, with the longer ones having a slight curvature. Bifurcation of the folds was observed. This division of folds he recognizes as indication of a developmental process. This thought he expresses as follows: "Wir halten es daher für möglich, dass die Leisten Histosysteme sind deren Anlage, im Laufe der Entwicklung durch spaltung vermehrt werden, so dass gelegentlich--bei unvollkommener Sonderung-Gabelungen entstehen."

Though familiar with Hermann's observations on the development of the papilla foliata in the rabbit, before our investigation was undertaken, it occurred to us that a careful study of the morphogenesis of the foliata area was still desirable, especially if free use was made of the Born wax plate reconstruction method, and a complete series of timed stages, ranging from the time of its first appearance as a definite area to the time of birth could be commanded. It seemed to us desirable to determine whether a definite pattern of epithelial folds was here present, whether the area had its anlage as whole or spread by peripheral growth with increase of the numbers of folds, as well as to determine more definitely the morphogenesis of the folds and the relations of the serous lingual glands to them. To do this it was found necessary to reconstruct by means of the Born plate method the

\footnotetext{
${ }^{9}$ Heidenhain, M., Untersuchungen über die Teilkörpernatur der Geschmacksknospen in der Papilla foliata des Kaninchens. Anat. Anz., vol. 45, 1914.

${ }^{10}$ Heidenhain, M. Über die Sinnesfelder und die Geschmacksknospen der Papilla foliata des Kaninchens. Beiträge zur Teilkörpertheorie, III. Arch. f. Mik. Anat., vol. 85, 1914.
} 
entire foliate area in a number of stages of development. The models here figured were prepared by Mr. Eggerth and constituted a part of the work required toward a Master of Arts degree with major in Anatomy.

All of the observations here recorded are based on timed material. For the 18, 19 and 20 day stages, frontal and sagittal series of heads of embryos rabbits were used, fixed in Carnoy's fluid and stained on the slide in iron hematoxylin and Congo red. For the stages covering 21 day to 30 day embryos, the lower jaw with tongue and larynx (for younger stages), the tongue and larynx (older stages) were removed and fixed in Yoshii's fluid. The material derived from the 21 day and 22 day embryos was sectional so as to obtain frontal sections of the tongue; approximately longitudinal sections of the foliata areas. For all other stages the foliate area with a block of underlying muscle was excised, oriented in the paraffine block so that longitudinal sections, at right angle to the main folds, might be cut. Complete series of sections, $4 \mu$ or $5 \mu$ in thickness of all stages, cut by the water-on-the-knife method with sliding microtome, were at our disposal. These were stained, some of the series in iron hematoxylin, others in Minot's hematoxylin and counterstained in Congo red. For complete series of relatively thin sections, the water-on-the-knife method with sliding microtome is especially recommended in preference to series cut on a rotary microtome, especially if the series are to be used, for reconstruction purposes. The models, though very time consuming in their preparation, presented no special difficulty in execution. They were made at a magnification of 300 diameters with thickness of the plates adjusted to meet the needs. The figures of the models, greatly reduced in reproduction, present the configuration of the epithelium of the foliate area as viewed from the under or mucosal side. A series of drawings of the epithelial border, taken from approximately the center of the papilla foliata of the several stages studied, from longitudinally cut series, is presented is figure 1. These figures may with profit be compared with the figure of the model for the equivalent stage, since for the stages modelled, the drawing for the respective 

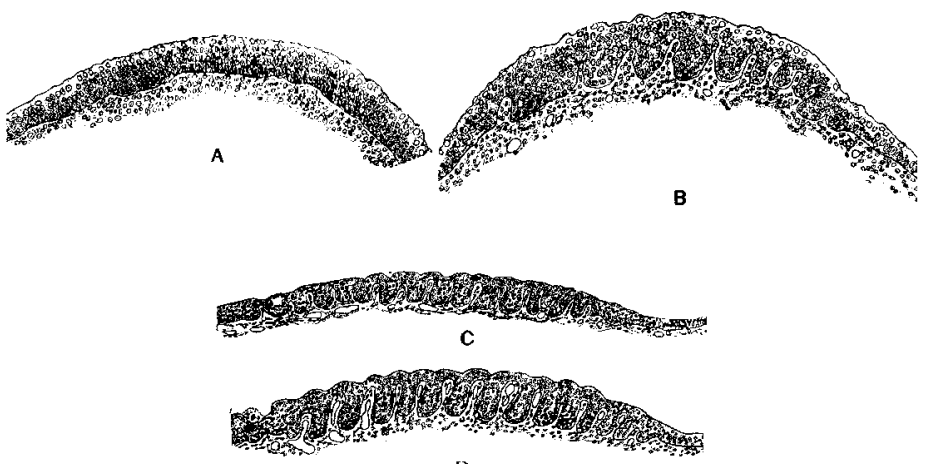

[)

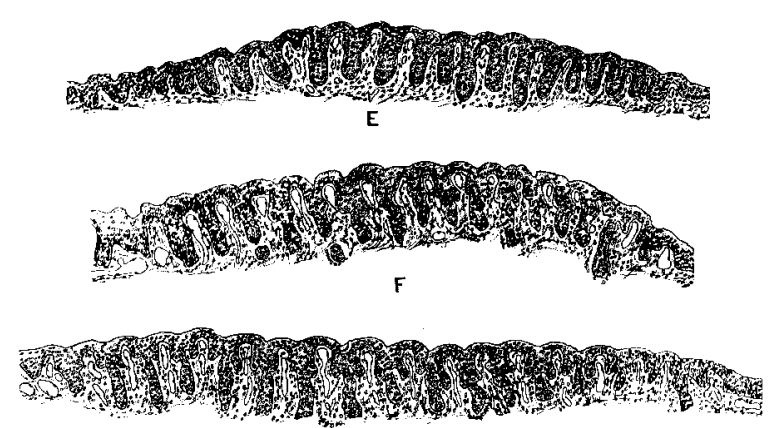

G
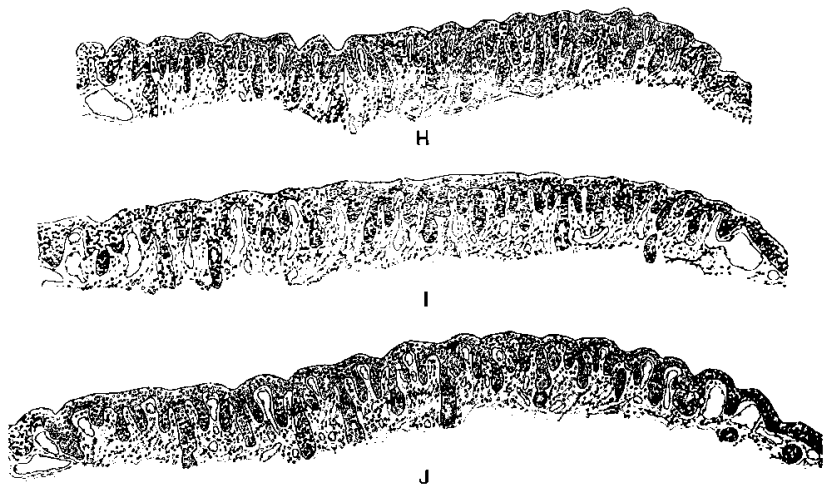

Fig. 1 Drawings of the epithelial border with the underlying mucosa of the foliate area of a rabbit embryos of different stages of development. $A$ and $B$, $\times 100 ; C$ to $J, \times 50$. A, from rabbit embryo of 18 days; $B, 20$ days; $C, 21$ days; $D, 23$ days; $F, 24$ days; $F$ ' 26 days; $G, 27$ days; $H, 28$ days; $I, 29$ days; $J, 30$ days. 
stage was taken from one of the sections of the series used in the preparation of the model covering that stage.

In presenting our own observations we will begin with the consideration of the stage in which the foliata area is first recognized and proceed in serial order of stages to the time of birth. It has appeared to us that the areas in which the foliate papillae develop, may be recognized before the appearance of the primary epithelial folds characterizing these areas. In a series of frontal sections, including the posterior portion of the tongue of a rabbit embryo of 18 days, a slightly elevated, oval area may be fairly clearly delimited on the lateral and dorso-lateral surface of the posterior part of the tongue, in front of the developing larynx and in front of the arcus palatoglossus of each side. In $A$, of figure 1 , there is presented a drawing of the epithelium of approximately the middle section passing through the right pre-foliate area. A study of this series permits the conclusion that the epithelium of the anterior part of this area is cut in fairly accurate cross-sections, while that of the posterior part is cut slightly obliquely. This area, as the figure may serve to show, is covered by squamous epithelium, which in the prefoliate area is slightly thicker than in the region anterior and posterior to this area. In a series of frontal sections of the tongue of a rabbit embryo of 20 days, the foliate area is clearly recognized, as may be observed from a study of section $B$, of figure 1 . The foliate areas present at this stage, on the under surface of the epithelium, a series of epithelial folds which clearly characterize these areas. The drawing was made from approximately the middle of the series of sections passing through the right foliate area of this stage. The section drawn presents a fairly accurate cross-section of the epithelium, but passes slightly obliquely through the area, not quite parallel to its long axis. That the 10 or 11 fairly well formed epithelial papillae, as seen in the sections, are in reality sections of epithelial folds is readily determined by graphic reconstruction and is corroborated by a study of a series of sagittal sections of the head of a rabbit embryo of 19 days in which the foliate areas are cut tangentially with reference to the epithelial surfaces, so that the epithelial folds are 
cut longitudinally for a short distance. The primary folds of the foliata areas, therefore, make their appearance about the 19th day of the rabbits' development and are clearly indicated on the under surface of the epithelium by the 20th day, although there is no definite indication of such epithelial folds on surface inspection, even when viewed with the aid of a stereoscopic binocular. The impression is gained that these folds spring into existence relatively suddenly as if by a folding of the under layer, the germinal layer, of the epithelium. As the figure may serve to show, the folding does not involve the entire thickness of the epithelium and there is observed no unusual number of mitotic figures in this foliate area. The mechanism we are unable to determine positively. In a study of the series of stages shown is figure 1 , it should be recognized that $A$ and $B$ of this figure were drawn at a magnification of 600 diameters, while all the other sections were drawn at a magnification of 300 diameters, the whole figure being reduced to the same extent in reproduction. For the next stage, that of a 21 day rabbit embryo, we are able to present a figure of a model showing the under surface of the epithelium one of the foliate areas as well as a drawing of one of the series of sections from which this model was made. In figure 2 is reproduced a photograph of the under or mucosal surface of a model representing a cast of the epithelium of the right foliate area and immediate surroundings, made at a magnification of 300 diameters. The right foliate area was cut from the tongue of a rabbit embryo of 21 days, oriented in the paraffine block so as to enable obtaining cross-sections of the major folds, shallow grooves on the surface, clearly evident under the stereoscopic binocular indicating the direction of these folds. From the finished reconstruction it is learned that the line of the sections is not parallel to the long axis of this foliate area. This is to be given cognizance in interpreting $C$, of figure 1. a drawing of the epithelium of one of the sections of this area, which does not present a section through its longest axis, though taken from about the middle of the series and as shown by the model, passes almost exactly at right angles to the long axis of the major folds. In figure 2 , the foliate area proper 
G. CARL HUBER AND ARNOLD H. EGGERTH
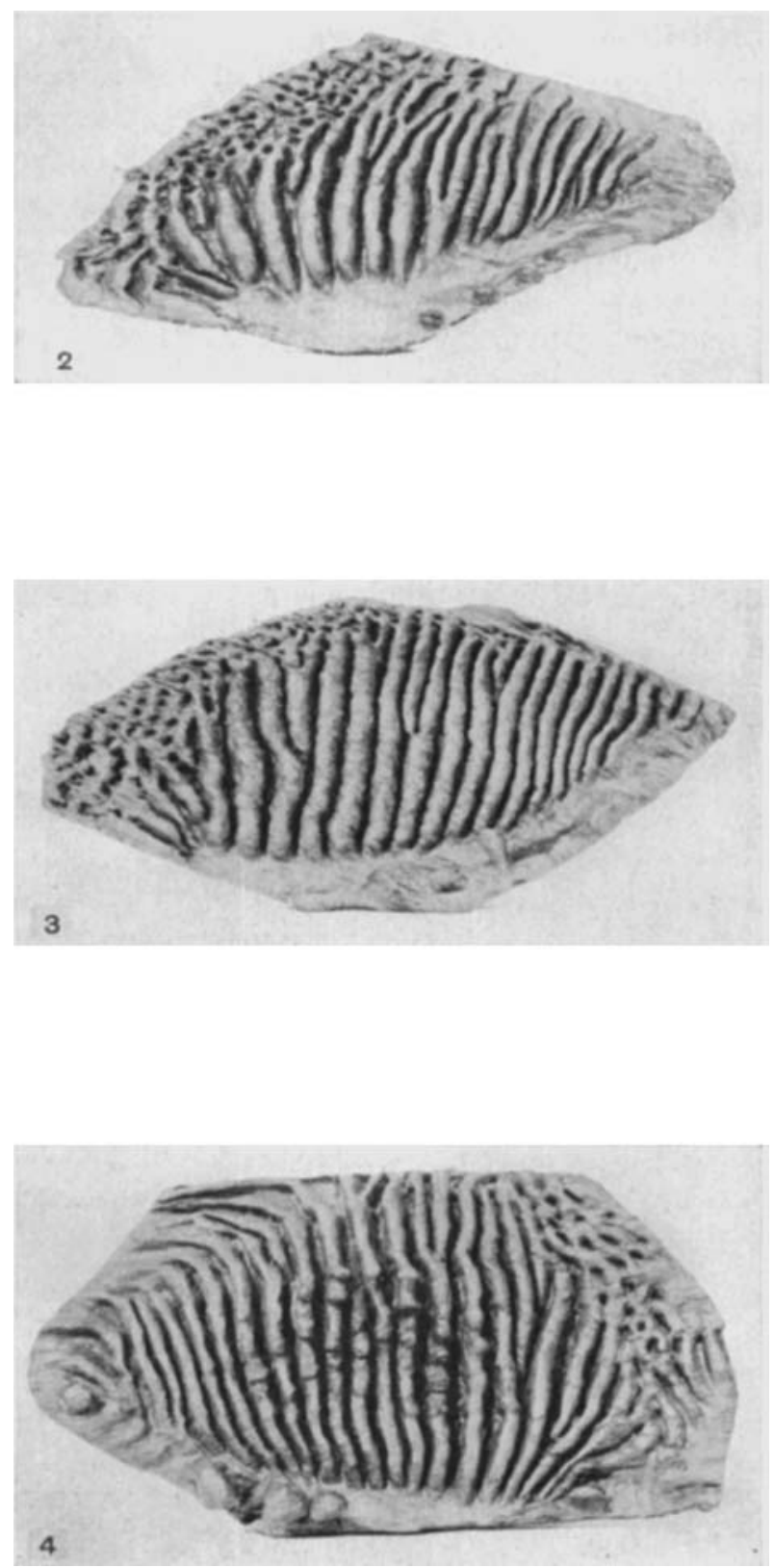
is readily delimited. The dorsal border of the area is directed upwards and the anterior border to the left. To the left and left upper portion of this figure the presence of epithelial pits, which received mucosal papillae may be observed, ventrally and posterior to the foliate area, the mucosal surface of the epithelium is fairly smooth. The foliate area as reconstructed presents an elongated egg-shape and in general contour, except for width, and in configuration is not unlike the foliate area of an adult rabbit as figured by Heidenhain ${ }^{10}$ in his text figures $d$, e and $f$. In this area, as the figure of the model may serve to show, there are present at least 15 primary epithelial folds. It may further be observed that there is at this stage division or bifurcation of certain of the primary epithelilal folds, both in a dorsal and in a ventral direction as also figured and discussed by Heidenhain, for the foliate area of the adult rabbit. The fact that about as many primary epithelial folds are present in the foliate area at this early stage in development as in the adult rabbit would seem to indicate that these primary epithelial folds may be regarded as 'Histosystems' in Heidenhain's terminology; further, the fact that about as much splitting or bifurcation of the primary epithelial folds is to be observed at this stage as in the adult rabbit may serve to indicate that the number of the primary epithelial folds is not materially increased by longitudinal splitting of folds of earlier stages of development. As may be observed from the figure, the primary epithelial folds converge toward about the middle of the ventral border, and the major folds present a slight curvature directed toward the front of the tongue, very much as described by Heidenhain

Fig. 2 Photograph of the mucosal surface of a wax plate reconstruction of the epithelium of the right foliate area of a rabbit embryo of 21 days. Magnification approximately 50 diameters.

Fig. 3 Photograph of the mucosal surface of a wax plate reconstruction of the epithelium of the right foliate area of a rabbit embryo of 23 days. Magnification approximately 50 diamteres.

Fig. 4 Photograph of the mucosal surface of a wax plate reconstruction of the epithelium of the left foliate area of a rabbit embryo of 24 days. Magnification approximately 50 diameters. Note the anlagen of the serous lingual glands in the center of the foliate area. 
for the adult rabbit. Section $C$, of figure 1, presents a drawing of the epithelium of approximately the middle of the series from which the model of figure 2 was made. The section is so placed that the epithelium bordering the anterior part of the foliate area is directed toward the left, as with all the sections of the series shown in figure 1. Perhaps 13 definite primary epithelial folds as seen in cross-section, may be observed in this figure. It should be observed that the shallow grooves, described as seen on surface view of this foliate area, fall over the center of the epithelial folds, and are the grooves into which empty the serous lingual glands in later stages of development. The primary mucosal folds interdigitate with the primary epithelial folds. The series of sections of the foliate areas of a rabbit embryo of 22 days are in all respects so similar to those of the 21 day stage just described, that a special consideration of the 22 day stage may here be dispensed with. The 23 day stage was reconstructed from an excellent series of sections which pass almost at right angle to the major primary epithelial folds and give the epithelium of this area in very good cross-section. The under surface of the model of the epithelium is presented in figure 3 , the accompanying section drawing in $D$, of figure 1 . Figure 3, thus shows the mucosal surface of the right foliate area of a rabbit embryo of 23 days, with the surrounding epithelium. There are found 16 well formed primary epithelial folds, three of which show division in a dorsal direction. These folds are well formed and clearly defined and are so clearly portrayed in the figure that a detailed consideration of them is deemed unnecessary. At the lower border of the figure there may be observed the anlage of a mucous gland, budding from the epithelium just ventral to the foliate area. Three such gland anlagen are observed in the model only one of which is seen in the figure. The mucous glands of the posterior and posterior ventral part of the rabbit's tongue thus have their anlagen at an earlier stage of development than do the serous lingual glands of the foliate area. When section $D$, of figure 1, (23 days) is compared with section $C$ (21 days) of this figure, it will be observed that the primary epithelial folds as seen in sections, are deeper in the older stage, 
though of essentially the same form. The primary mucosal folds are likewise better developed in the older stage. It may be observed from a study of the figure that nearly every primary mucosal fold carries near its crest a capillary loop, which capillary loops, for relatively long distances, course parallel to the epithelium. We have included a reconstruction and drawing of a section of a foliate area of a rabbit embryo of 24 days, in that in this stage there is observed the first indication of the anlagen of the serous lingual glands also characteristic changes in the primary epithelial folds are seen. In figure 4, is reproduced the mucosal surface of a reconstruction of the epithelium of the left foliate area of a rabbit embryo of 24 days. (In comparing figures 4,5 and 6 , with the figures 2 and 3 , it will be noted that figures 2 and 3 , are of the right foliate area, while the other three figures, 4, 5 and 6 , are of the left area. The pitted under surface of the epithelium, mucosal pipillae, indicates the region anterior to the foliate area. In the sections of all the stages figured in figure 1, the anterior border is to the left of the figure, so as to make a comparison of sections more easy.) This area present perhaps 16 primary folds, several divided folds may be seen, one of which divides ventrally. The general arrangement of the primary epithelial folds is clearly indicated in the figure. Especial attention is drawn to the serated edge of the middle portions of the longer, centrally placed folds. The folds, in this region present on their crests short bud-like protrusions recognized as anlagen of the serous lingual glands. As is evident from the figure, these glands appear first in the more central region of the foliate area. Hermann has previously stated that the central region of a foliate area is most fully developed. To the right and ventral in this figure there may be observed the anlagen of several mucous glands, distinctly longer than the serous lingual glands, presenting bulbous ends, having an epithelium which enables their recognition as mucous glands. In several of the more centrally placed primary epithelial folds, as seen in the reconstruction, there may be observed about midway down the respective fold, a narrow shelf passing along each side of the respective fold. These represent the first indication 

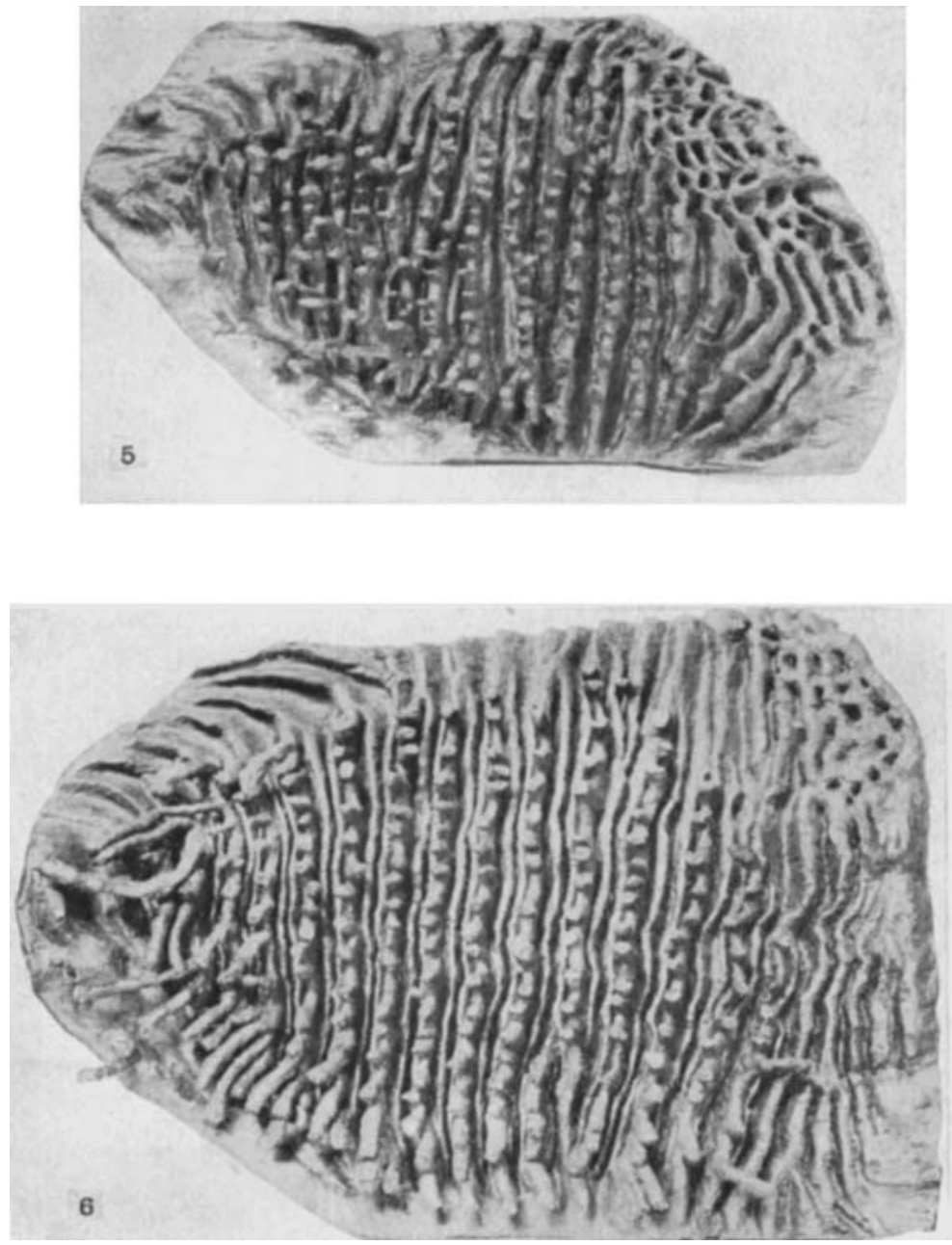

Fig. 5 Photograph of the mucosal surface of a wax plate reconstruction of the epithelium and ducts of the serous lingual gland of the foliate area of a rabbit embryo of 26 days. Magnification approximately 50 diameters.

Fig. 6 Photograph of the mucosal surface of a wax plate reconstruction of the epithelium and ducts of the serous lingual glands, with the primary and secondary epithelial folds of the foliate area of a rabbit embryo of 30 days. Magnification approximately 50 diameters. 
of the secondary epithelial folds and are much more clearly discerned in the drawing of one of the sections of the series from which this model was made, $E$, of figure 1 . In this section, the epithelium bordering the anterior part of the foliate area is directed toward the left while in the model, this region, recognized by the epithelial pits, is directed towards the right. This series of sections is cut almost parallel to the long axis of the foliate area and present good cross-sections of the epithelium of the more central part of the field. It will be observed on comparison with the former sections, that the primary epithelial folds are deeper than in earlier stages and that they are thicker and broader in their basal portions, this thickening extending to about half the depth of the respective epithelial fold. The lower border of this thickening on each side of a primary epithelial fold marks the anlagen of the secondary epithelial folds, much more clearly seen in older stages. It may be observed that certain of the primary epithelial folds extend for a greater distance into the mucosa than to others. These longer 'folds' indicate regions in the prepanation in which the plane of section included serous lingual gland anlagen. The two series of sections of the 25 day stage were both cut quite obliquely to the long axis of the respective foliate areas, and thus also the primary epithelial folds, giving these folds a broader contour than is warranted from a study and part graphic reconstruction of these series. A figure of this stage is not included. This stage shows only a very slight advance in development over that shown for the 24 day rabbit embryo. The stage of the 26 day rabbit is presented both in model and in section. The model reproduced in figure 5 , is of the left foliate area of a 26 day rabbit embryo. There are present in all 15 well developed primary epithelial folds and two less fully developed ones. The secondary epithelial folds are more fully developed, extending deeper into the mucous, than in the 24 day stage, though these secondary epithelial folds are somewhat difficult to discern in the figure of the model in that they are hidden by reason of the compactness of the folds; on study of the model itself, they are clearly recognized and may be seen in the section of this 
stage to be discussed presently. The serous lingual glands show marked increase in length, many having pierced the musculature of the tongue for quite a distance, having bulbous, but as yet unbranded ends. There is also observed a marked increase in the number of the glands, no less than 140 gland ducts are to be counted in the reconstruction of this area. The ducts of these glands arise from the crests of the primary epithelial folds. A number of large mucous glands are evident to the left and lower left in the figure, just outside of the folds limiting the foliate area. A typical section of the epithelium, taken approximately from the middle of the series from which the model was made is shown in drawing $F$, of figure 1 . The thickened bases of the primary epithelial folds, with distinct indications of the seeondary epithelial folds, are easily recognized in this figure. In this figure many of the primary epithelial folds extend as gland ducts into the mucosa, and certain ones beyond the limits of the drawing, which includes the mucosal layer to the upper level of the tongue musculature. It is requested to note the positions of the capillary loops in the crests of the primary mucosal folds as seen in figure $1, F$, and compare the same with the positions of the capillary loops in the crests of the simpler mucosal folds as seen in figure $1, D$. The relative position of these capillary loops remains constant and thus enable an interpretation of the morphogenesis of the epithelial and mucosal folds. In the rabbit embryo of 26 days the foliate areas are readily recognized with the unaided eye. Since, as may be observed from a study of the section as drawn in $F$, of figure 1 , the surface grooves have acquired depth, giving the upper border of the section a distinctly scalloped appearance; it will also be observed that these surface grooves are situated over the middle of the primary epithelial. folds from the crests of which bud the serous lingual glands. Drawings $G, H$ and $I$, of figure 1 , are from series of sections of the foliate areas of rabbit embryos of 27,28 and 29 days, respectively. They are inserted mainly to give details of the development of the secondary epithelial and the secondary mucosal folds, developing - the epithelial folds -from the bases of the primary epithelial folds with which they run parallel. In each figure 
the positions of the capillary loops is to be noted. It will be seen that the secondary epithelial folds are clearly indicated in $G$, of figure 1, they are longer and more distinct in $H$, of this figure and in section $I$, in figure 1 , appear in cross-sections as distinct, slender papillae, diverging slightly from each side of the bases of the several primary epithelial folds; these slender papillae representing cross-sections of the secondary epithelial folds. The serous lingual glands have acquired length during these stages extending now deeply into the tongue musculature, showing bulbous or branched ends and evidence of the presence of a lumen in many of them. In figure 6 , is shown a reconstruction of the left foliate area of a rabbit embryo of 30 days, removed from the uterus just prior to birth. It represents the oldest stage modelled and gives the mucosal view of the epithelium of a foliate area which in many respects is like that of a foliate area of an adult rabbit. This area presents 16 primary folds and toward the right of the figure several small folds which have the same general directions as do the foliate folds, but without presenting their morphology in that they are not accompanied by secondary folds and have not given origin to serous lingual glands. This area has spread out to such extent, in course of development, that the secondary epithelial folds are clearly seen, as running parallel to each side of the respective primary epithelial folds. The positions of the primary and secondary mucosal folds can easily be depicted in that they fill the clefts bounded by the epithelial folds. Approximately 200 ducts of serous lingual glands are counted in this reconstruction, probably about the number present in a foliate area of the adult rabbit. The backward turn of the ducts of the serous lingual glands of the most posterior portion of the foliate area, to the left in figure 6 , is to be noted. At this stage the ducts lead to the surface of the epithelium and do not empty at the bottom of the deep clefts as found in the grown and fully developed area. It seems reasonable to conclude although we have not at our disposal the necessary stages to substantiate this conclusion, that the deep clefts separating the surface folds of the fully developed foliate area, are in part formed through a desquamation of the 
epithelium separating the duct lumina as they pass through the epithelium to the surface. In section $J$, of figure 1 is given a drawing of the epithelial border and underlying mucosa of approximately the middle section of the series from which the model in figure 6 was made. This section is in direction nearly parallel to the long axis of the foliate area sectioned and cuts the major folds at right angle, presenting also a good cross-section of the epithelium. This figure is in a large measure self explanatory, primary and secondary epithelial and mucosal folds are clearly discernible as are also the ducts of the serous lingual glands. It should be noted that in the fully developed foliate area, the capillary loops found in the crests of the primary mucosal folds, separating the primary epithelial folds, $D$ and $E$, of figure 1 fall to the center of the surface folds as found in the fully developed area foliata, the clefts separating these surface folds falling in line with the rows of ducts of the serous lingual glands as seen in figure 6 , each such surface fold embracing the tissue found between two rows of ducts of the lingual serous glands.

The question of the histogenesis of the gustatory buds has not formed a special portion of this investigation. The methods of fixation and staining used, have enabled us to confirm in a large measure the early observations of Hermann ${ }^{3}$ as concerns the development of the gustatory buds of the rabbit's foliate area. Relatively few gustatory buds are present in the foliate areas of the rabbit at the time of birth. These few are nearly all found scattered here and there near the mouths of the ducts of the serous lingual glands. A study of the histogenesis of the gustatory buds of the foliate areas of the rabbit is projected in a future investigation, with the aid of special technical methods, of which the pyridine silver impregnation method shall receive special consideration. It seemed to us advisable to first acquire familiarity with the morphogenesis of the foliate area, before a study of the histogenesis of the gustatory buds was undertaken. The severance of official connection with the Department of Anatomy of one of us has prompted us to place on record the results of this investigation as thus far attained, namely, the 
morphogenesis of the foliate areas of the rabbit from time of its first appearance to the time of birth. Our observations may be summarized briefly as follows:

1. The foliate areas of the rabbit's tongue may be recognized as slightly elevated, oval pre-foliate areas in rabbit embryos of the 18th day.

2. The primary epithelial folds characterizing these areas, begin to appear on the 19th day, and are well formed and present in approximately full number on the 21 st day. These primary epithelial folds are to be recognized as 'histosystems,' since they are found in fairly constant number throughout the period of morphogenesis of these areas; practically the entire foliate area on each side being in anlage at the same time.

3. The serous lingual glands, associated with the foliate areas, make their appearance, in the central region of the foliate area, during the 24th day. The secondary epithelial are in anlage during the 24th day, in the central region of foliate area, and show appreciable development over the entire area by the 26th day.

4. The epithelial and mucosal folds, both primary and secondary, as viewed from the under side of a foliate area of the 30th day, show an arrangement and state of development which is essentially the same as in a fully developed foliate area, although the deep clefts or grooves which separate the surface folds of the adult area are as yet merely indicated. At this period of development the gustatory buds are as yet few in number and are found in close relation to the mouths of the ducts of the serous lingual glands, which reach the surface of the epithelium. 